\title{
Zeitverlauf der Änderungen an energiereichen Phosphorverbindungen im Meerschweinchenherzen nach Asphyxie und Wiederbeatmung
}

\author{
Kurt Moser \\ I. Medizinische Universitätsklinik, Wien, Österreich
}

\begin{abstract}
Time course of changes in energy-rich phosphate compounds in the heart of the guinea pig following asphyxia and reanimation. In the myocardium of guinea pigs the levels of ATP, ADP, AMP, creatine phosphate and inorganic phosphate, respectively, were determined following different periods of asphyxia and subsequent reanimation. Depending upon the duration of asphyxia, all energy-rich phosphate compounds decreased, creatine phosphate demonstrating this effect most markedly. Following reanimation, the energy-rich phosphate compounds underwent partial or complete regeneration, unless asphyxia lasted longer than 5 to 7 minutes.
\end{abstract}

\section{EINLEITUNG}

Für einen geregelten Stoffwechsel des Herzmuskels ist eine genügende Versorgung mit Sauerstoff, also eine gute Coronardurchblutung erforderlich. Ein erhöhter Satuerstoffbedarf des Herzens wird durch Mehrdurchblutung des Myocards befriedigt. Da nun die energiereichen Phosphatester des Herzmuskels hauptsächlich durch den oxydativen Stoffwechsel regeneriert werden, würden sie bei Hypoxie starken Veränderungen unterliegen. Wir haben zu verschiedenen Zeitabständen einer Asphyxie ihr Verhalten im Herzmuskel des Meerschweinchens untersucht.

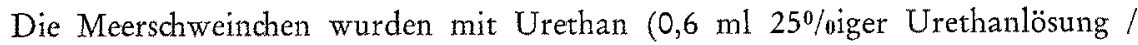
100 g i. p.) narkotisiert und mit der Beatmungspumpe nach ScHuler künstlich beatmet. 15 Minuten nach Exöffnung des Thorax wurde bei den Kontrolltieren das Herz mit einem Scherenschlag entfernt und sofort in flüssiger Luft eingefroren. Bei den Versuchstieren wurde nach Eröffnung des Thorax die Atmung durch Abklemmung der Trachea zu verschiedenen Zeiten unterbrochen und nach verschieden langer Dauer der Asphyxie das Herz entfernt. Im fixierten Herzmuskel wurde Adenosintriphosphorsäure (ATP), Adenosindiphosphorsäure (ADP), Adenosinmonophosphorsäure (AMP), Kreatinphosphat $(\mathrm{CP})$ und Orthophosphat $\left(\mathrm{P}_{\mathrm{a}}\right)$ bestimmt. Hierzu wurde die chromatographische Methode von Fleckenstein \& Gerlach (1953), Fleckenstein \& Janke (1953), Gerlach et al. (1957) sowie Gerlach et al. (1958) verwendet. 


\section{ERGEBNISSE}

Bei Kontrolltieren finden sich 15 Minuten nach Eröffnung des Thorax und bei künstlicher Beatmung im Mittel 3,56 $\mu \mathrm{Mol} / \mathrm{g}$ Gewebe ATP, 1,07 $\mu \mathrm{Mol} / \mathrm{g}$ Gewebe ADP, 0,77 $\mu \mathrm{Mol} / \mathrm{g}$ Gewebe AMP, 7,04 $\mu \mathrm{Mol} / \mathrm{g}$ Gewebe CP und 1,82 $\mu \mathrm{Mol} / \mathrm{g} \mathrm{Ge}-$ webe $P_{\mathrm{a}}$. Nach einer Asphyxiedauer von 1, 2 oder 3 Minuten kommt es zu einem laufenden Abfall der CP-Werte und zu einem steten Anstieg von anorganischem Phos-

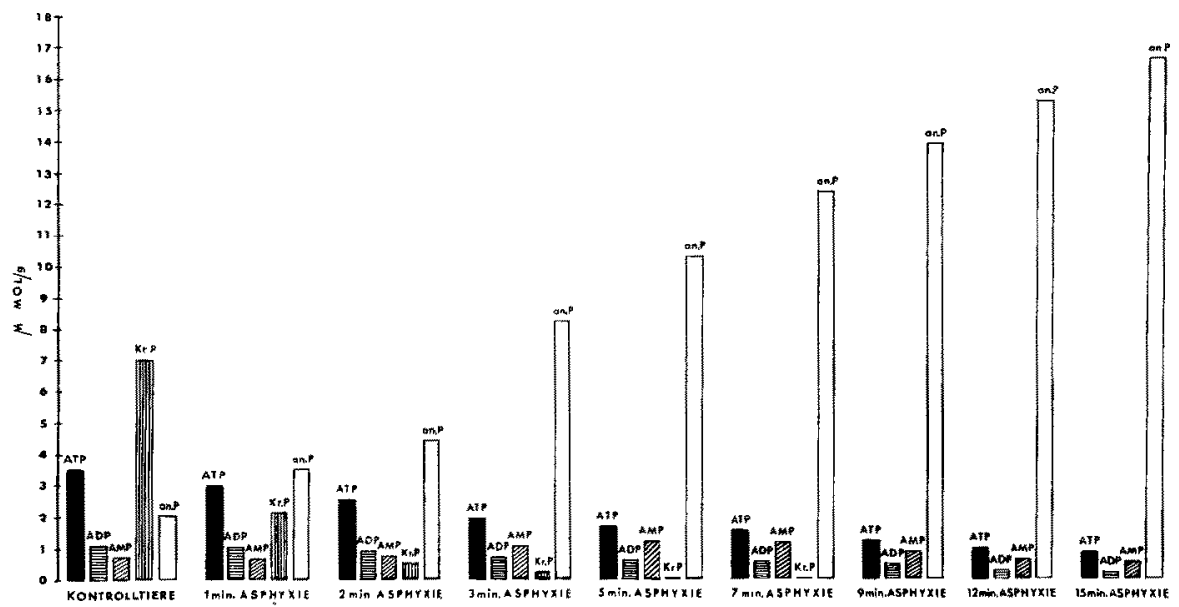

Abb. 1: Einfluß einer yerschieden langen Dauer von Asphyxie im Meerschweinchenherzen auf die Konzentration von ATP, ADP, AMP, Kreatinphosphat und Orthophosphat

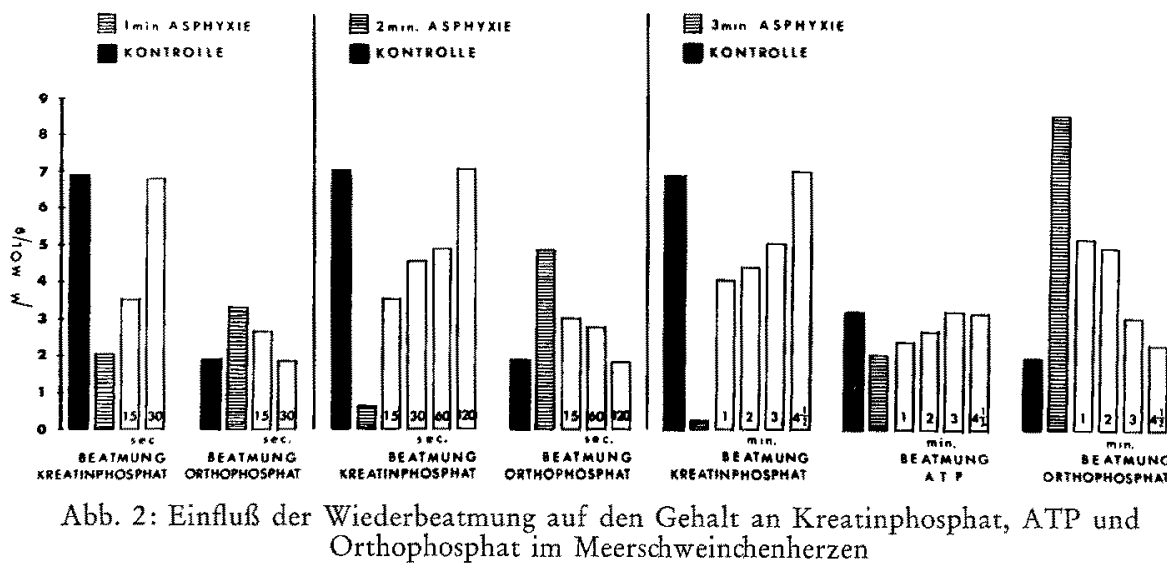

phat. Bei einer Asphyxiedauer von 5 Minuten war CP nicht mehr nachweisbar. Die ADP- und AMP-Werte zeigen erst 2 Minuten nach Asphyxie eine Anderung, wobei ATP, ab der dritten Minute auch ADP, bis zur 15. Minute Asphyxie einen laufenden Abfall zeigen, während AMP bis zur fünften Minute ansteigt und erst danach abfällt (Abb. 1). 15 Minuten nach Asphyxie war im EKG entweder schon ein Kammerflimmern 


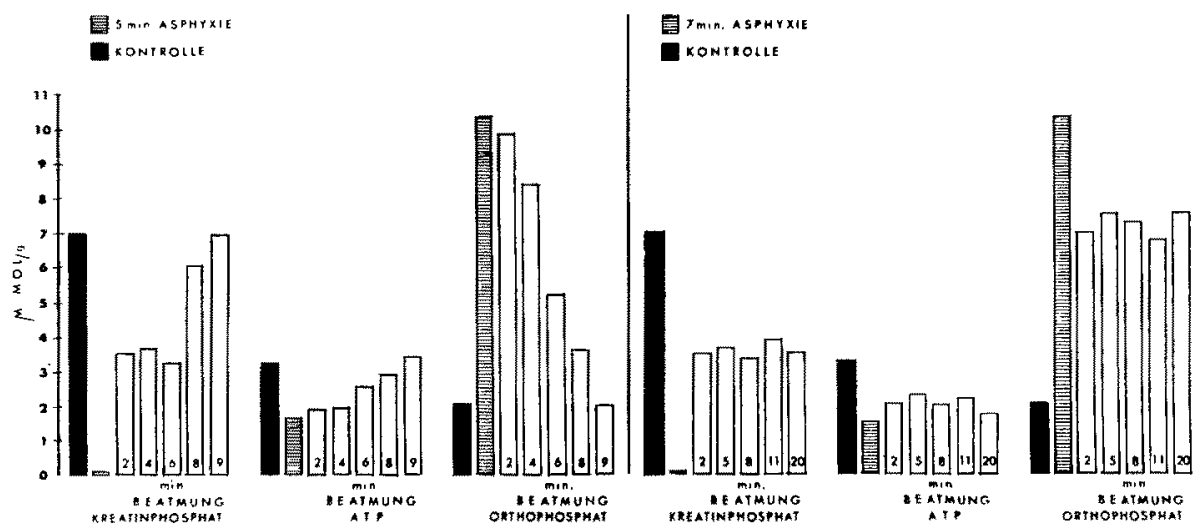

Abb. 3: Einfluß der nach fünf und sieben Minuten dauernden Asphyxie und anschließender Wiederbeatmung auf den Gehalt an Kreatinphosphat, ATP und Orthophosphat im Meerschweinchenherzen

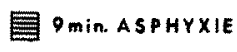

KONTROLLE
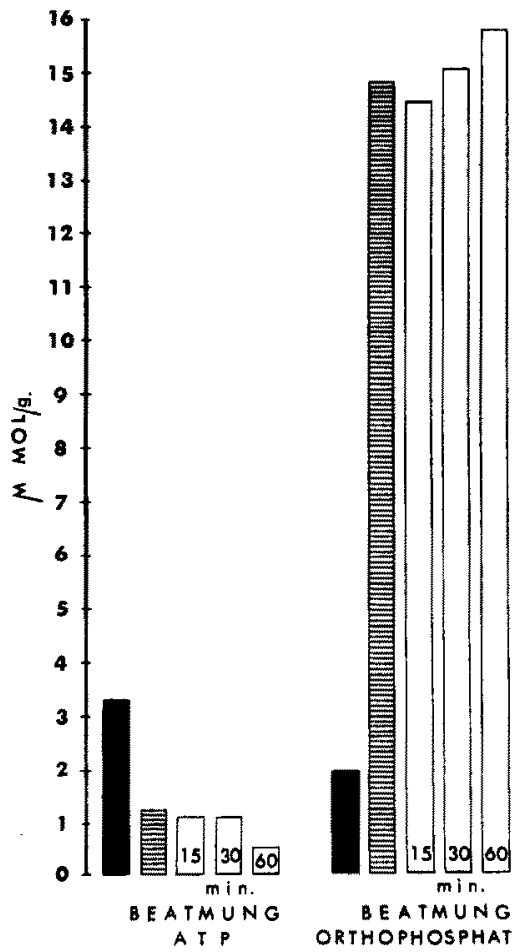

Abb. 4: Die nach neun Minuten Asphyxie beobachteten ATP- und Orthophosphatkonzentrationen mit anschließender Wiederbeatmung im Meerschweinchenherzen 
oder eine hochgradige Bradycardie mit schweren EKG-Veränderungen zu beobachten. Bei einigen Herzen war trotz elektrischer Herztätigkeit eine Asystolie feststellbar.

In einer weiteren Versuchsreihe (Abb. 2,3 und 4) werden nach verschieden langer Dauer der Asphyxie die Tiere wieder beatmet. Nach Asphyxiedauer von 1 Minute normalisiert sich das CP, wenn eine Wiederbeatmung von einer halben Minute durchgeführt wurde. Bei 2 Minuten Asphyxie benötigt die Wiederherstellung des CP 2 Minuten Wiederbeatmung. Die EKG-Veränderungen, die nach 2 Minuten Asphyxie aufgetreten waren (Rhythmusstörungen, verursacht durch AV-Blockierungen) konnten bereits nach einer kurzen Wiederbeatmung (10 bis 20 Sekunden) zum Verschwinden gebracht werden. Zugleich war auch eine 50- bis 60prozentige Regeneration des CP nachweisbar. Dauert die Asphyxie 3 Minuten, so ist bereits eine Wiederbeatmung von 41/2 Minuten für die Wiederherstellung von normalen ATP- und CP-Werten notwendig. Bei einer Asphyxiedauer von 5 Minuten sind bereits 9 Minuten erforderlich; bei einer Asphyxiedauer von 7 Minuten sind trotz einer Wiederbeatmung von 20 Minuten keine annähernden Normalwerte von ATP und CP zu erreichen, obwohl im EKG bereits nach 1 bis 2 Minuten Wiederbeatmung Normalrhythmus zu beobachten ist. Die nach 9, 12 und 15 Minuten Asphyxie beobachteten schweren Stoffwechselveränderungen können durch Wiederbeatmung nicht mehr beeinflußt werden. Die zugleich auftretenden schweren EKG-Veränderungen (Kammerflimmern, Extrasystolie, AV-Blockierung und intraventrikuläre Leitungsstörangen) können ebenfalls durch eine Wiederbeatmung nicht geändert werden.

\section{DISKUSSION}

Die von uns im ungeschädigten Meerschweinchenherzen bei künstlicher Beatmung gefundenen Werte der säurelöslichen Phosphorverbindungen entsprechen größenordnungsgemäß den von HochreIN \& Döring (1958) angegebenen. FLECKENSTEIN (1958) konnte in experimentellen Untersuchungen an Ratten und Katzen nachweisen, daß bei Anoxie und bei akutem Coronarverschluß eine Senkung der Vorräte an ATP und CP im Herzmuskel eintritt. In weiteren Anoxieversuchen konnten DörING \& GERLACH (1957) im Rattengehirn zeigen, daß es zuerst zu einem Abfall von $C P$ und dann von ATP bei gleichzeitigem Anstieg von $P_{a}$ kommt. Auch wir konnten in unseren Versuchen bei Asphyxie einen raschen Abfall von $\mathrm{CP}$ im Myocard des Meerschweinchens beobachten. Dieser rasche Abfall von $C P$ und später von ATP hat seine Ursache in der hohen Empfindlichkeit des Herzmuskels gegenüber Sauerstoffmangel, welche die des Skelettmuskels um ein Vielfaches übersteigt. Dies konnte auch FECKENSTEIN et al. (1959) durch die Aufnahme von mit ${ }^{32} \mathrm{P}$ markiertem Orthophosphat in die ATP- und CP-Fraktion des M. quadriceps der Ratte zeigen, die etwa 20mal langsamer als in die entsprechende Fraktion des Rattenherzmuskels erfolgt. Daraus ist ersichtlich, daß das gesamte Niveau der oxydativen Phosphorylierung im Myocardgewebe beträchtlich höher liegt als in der Skelettmuskulatur. Wenn nun das Sauerstoffangebot in der Asphyxie wegfällt, wird die oxydative Phosphorylierung zum Erliegen kommen. Dies wird mit einem Abfall der energiereichen Phosphatester im Herzmuskel gekoppelt sein. Einerseits wird die Glykolyse allein nicht imtsande sein, für längere Zeit einen genügend großen Anteil der energiereichen Phosphatverbindungen zu bilden, da das Ver- 
hältnis der ATP-Ausbeute von Atmung zu Glykolyse 1:19 beträgt. Andererseits wird das Herz auch die ihm zu Verfügung stehenden Substrate in der Asphyxie rasch glykolytisch umsetzen und dadurch an Substrat verarmen. Der rasche und intensive Abfall von CP bereits nach einer Minute Asphyxie ist damit zu erklären, daß CP als schnell verfügbare Reserve in der Notfallssituation des Herzens eingesetzt werden kann. Nach einer Minute Asphyxie haben wir noch keinen sicheren Abfall der ATP-Konzentration im Herzmuskel nachweisen können. Dies könnte man darauf zurückführen, daß durch den intensiven Abbau von CP unter Sauerstoffmangel genügend Energie frei wird, um die durch Asphyxiè ausgefallene oxydative Phosphorylierung und damit stark verzögerte Regeneration von ATP noch ausgleichen zu können. Im weiteren Verlauf des Versuches fällt diese Energiereserve aus, und es kommt zu einem Abfall von ATP und ADP sowie zum Anstieg des Orthophosphats. Nach 15 Minuten Asphyxic fanden wir trotz Asystolie, Kammerflimmern oder auch hochgradiger Bradycardie mit schwersten EKG-Veränderungen noch einen vergleichsweisen hohen ATP-Wert von $0,8 \mu \mathrm{Mol} / \mathrm{g}$ Gewebe. Dieser hohe Wert wird zum Teil aus dem Exhaltungsstoffwechsel von überlebenden Myocardzellen und vielleicht auch teilweise von den im Herzmuskel zurückgebliebenen Erythrozyten, die einen ausgeprägten glykolytischen Stoffwechsel haben, herrühren.

Aus unseren Versuchen ist ersichtich, daß nach einer Asphyxiedauer von 1 bis 5 Minuten in einer relativ kurzen Zeit nach Wiederbeatmung die oxydative Phosphorylierung in Gang gekommen ist und wieder normale Verhältnisse der energiereichen Phosphorverbindungen eintreten. Nach einer Asphyxie von 7 Minuten Dauer müssen auch beim schlagenden Meerschweinchenherzen bereits irreversible Schäden in der oxydativen Phosphorylierung eingetreten sein. Aus unseren Versuchen ergibt sich auch die nicht uninteressante Beobachtung, daß zum Zeitpunkt des Eintretens von Normalrhythmus im EKG nach Wiederbeatmung die Kreatinphosphatmenge zu 50 bis $60 \%$ wieder regeneriert war. In Entsprechung zu diesem Befund konnte FleckensteIN (1958) die Beobachtung machen, daß es im Meerschweinchenherzen bei einem Verlust von $2 / 3$ bis $3 / 4$ der Kreatinphosphatmenge zum Auftreten von Insuffizienzerscheinungen kommt. Man könnte sich auch vorstellen, daß bei der Wiederbeatmung nach Oberwindung dieser Herzinsuffizienz und bei Wiedereinsetzen des oxydativen Stoffwechsels die in der Glykolyse vermehrt auftretenden und sich anhäufenden Zwischenprodukte beseitigt werden. Diese Intermediate fallen dann vielleicht als ektopische Reizbildner aus; aus; es kommt zu diesem Zeitpunkt zum Normalrhythmus und daher zur relativ hohen CP-Menge von 50 bis $60 \%$ des Normalwertes. Wiederbeatmungsversuche nach 9,12 und 15 Minuten Asphyxiedauer konnten weder in den schweren EKG-Veränderungen noch in dem Gehalt der Nucleotide eine Anderung herbeiführen. Nach dieser Asphyxiedauer sind demnach beim thorakotomierten Meerschweinchen mit schlagendem Herzen schon schwerste Stoffwechselveränderungen vor sich gegangen, so daß die Wiederbeatmung nicht mehr in der Lage war, die oxydative Phosphorylierung in Gang zu bringen. Nach diesem Zeitraum wird außer der Asphyxie auch noch die Herzischämie eine Rolle spielen, da die Insuffizienz, die bereits nach ein bis zwei Minuten Asphyxiedauer beginnt, ein schweres Kreislaufversagen auslösen wird. Diese Untersuchungen, dies möchte ich noch besonders hervorheben, beziehen sich auf Meerschweinchen, die thorakotomiert wurden und deren Herz in der Asphyxie Kreislaufarbeit zu leisten 
hatte. Bei Anoxieversuchen mit stillgestelltem Herzen, Untersuchungen die noch im Gange sind, werden zeitlich andere Werte zu erwarten sein.

\section{ZUSAMMENFASSUNG}

1. Nach verschieden langer Dauer von Asphyxic und anschließender Wiederbeatmung wurde im Meerschweinchenherzen ATP, ADP, AMP, Kreatinphosphat und anorganisches Phosphat bestimmt.

2. Bereits 1 Minute nach Asphyxie kam es zu einem deutlichen Abfall von Kreatinphosphat und Anstieg von anorganischem Phosphat; 5 Minuten nach Asphyxie war Kreatinphosphat nicht mehr nachweisbar.

3. ATP fiel 2 Minuten nach Asphyxie ab und zeigte einen weiteren Abfall bis $15 \mathrm{Minu}-$ ten nach Asphyxie, während AMP zuerst einen Anstieg, später einen Abfall zeigte.

4. Bei Asphyxiedauer von 1 Minute regenerierten sich die energiereichen Phosphorverbindungen im Meerschweinchenherzen nach Wiederbeatmung von 1/2 Minute und bei 5 Minuten Asphyxiedauer nach 9 Minuten Wiederbeatmung.

5. Dauert die Asphyxie 7 Minuten, so ist keine vollständige Regeneration der energiereichen Phosphorverbindungen mehr erreichbar.

6. War bei der Wiederbeatmung eine 50- bis 60 prozentige Regeneration des Kreatinphosphatanteiles eingetreten, so verschwanden im EKG regelmäßig die Rhythmusstörungen.

\section{ZITIERTE LITERATUR}

Döring, J. H. \& GerLach, E., 1957. Säurelösliche Phosphorverbindungen des Gehirns unter dem Einflußs von Anoxie, Ischämie und narkotischen Stoffen. Arch. exp. Path. Pbarmak. $232,271-273$.

FleCKensteIn, A. \& GerLaCH, E., 1953. Papierchromatographische Trennung von Adenosintriphosphat, Adenosindiphosphat und anderen Phosphorverbindungen bei Säugetier- und Tauben-Erythrozyten verschiedenen Kaliumgehaltes. Arch. exp. Patb. Pharmak. 219, $531-548$.

- \& JANKe, J., 1953. Papierchromatographische Trennung von ATP, ADP und anderen Phosphorverbindungen im kontrahierten und erschlaffen Froschmuskel. Pflïgers Arch. ges. Physiol. 258, 177-199.

- 1958. Der Herzmuskel und seine Abhängigkeit von der Koronardurchblutung. Wiener Z. innere Med. 39, 69-83.

- JANKE, J. \& GerLACh, E., 1959. Konzentration und Turnover der energiereichen Phosphate des Herzens nach Studien mit Papierchromatographie und Radiophosphor. Klin. Wrschr. 37, 451-460.

Gerlach, E., Fleckenstein, A. \& Freundt, K. J., 1957. Konzentration und Turnover der Adenosin- u. Guanosin-Phosphate sowie anderer säurelöslicher Phosphorverbindungen in Taubenerythrozyten. Pflügers Arch. ges. Pbysiol. 263, 682-703.

- WEBER, E. \& DöRING, H. J., 1955. Einige Lösungsmittel für die Papierchromatographie von Phosphorsäureestern. Arch. exp. Path. Pharmak. 226, 9-17.

Hochrein, H. \& Dörnng, H. J., 1958. Das Verhalten der energiereichen Phosphatverbindungen des Myocards im Normalzustand und bei experimenteller Insuffizienz durch Phenylbutazon. Pfiügers Arch. ges. Physiol. 267, 313-330. 
Diskussion im Anscbluß an den Vortrag Moser

BURMEISTER: Idh möchte fragen, ob die geschilderten Beobachtungen auch an jungen Tieren gemacht wurden?

Mosen: Wir haben alle Versuche an gleichaltrigen, adulten Tieren durchgeführt. 